\title{
Constraining the environment of GRB 990712 through emission line fluxes ${ }^{\star}$ (Research Note)
}

\author{
A. Küpcü Yoldaşs ${ }^{1}$, J. Greiner ${ }^{1}$, and R. Perna ${ }^{2}$ \\ 1 Max-Planck-Institut für extraterrestrische Physik, Giessenbachstrasse 1, 85748 Garching, Germany \\ e-mail: ayoldas@mpe.mpg.de \\ 2 JILA and Department of Astrophysical and Planetary Sciences, University of Colorado, 440 UCB, Boulder 80309, USA
}

Received 23 May 2006 / Accepted 27 June 2006

ABSTRACT

\begin{abstract}
Aims. The energy output in the gamma-ray burst (GRB) prompt emission and afterglow phase is expected to photoionize the surrounding medium out to large radii. Cooling of this gas produces line emission that is particularly strong in the optical, whose variability is a strong diagnostics of the gas density and geometry in the close environment of the burst.

Methods. We present the results of a spectral time-series analysis of the host galaxy of GRB 990712 observed up to 6 years after the burst. We analyze the emission line fluxes, together with those of the previous observations of the same GRB, in search of photoionization signatures.

Results. We find that the emission line fluxes show no variation within the uncertainties up to 6 years after the burst, and we use the measured line intensities to set a limit on the density of the gas within a few parsecs of the burst location. This is the first time that emission from cooling GRB remnants is probed on time scales of years.
\end{abstract}

Key words. gamma rays: bursts - stars: circumstellar matter

\section{Introduction}

In the fireball model of GRBs (e.g. Rees \& Meszaros 1992), the energy released from the collapse of a massive star is converted into kinetic energy of thin baryonic shells that expand at ultra-relativistic speeds. After producing the prompt $\gamma$-ray emission by internal shocks between different shells, the residual hits the surrounding gas and drives an ultra-relativistic shock into the ambient medium. The shock accelerates relativistic electrons leading to the observed X-ray to radio afterglow radiation through synchrotron emission. The emission of the X-ray afterglow, integrated over the first 7-10 days, typically contains the same energy as the primary $\gamma$-ray burst itself (Rees \& Meszaros 1998).

Whatever the density of the GRB environment, photoionization of the circumburst material both by the prompt X-ray emission and by the X-ray and UV afterglow emission will be inevitable. If the circumburst density is high, it will lead to timedependent (on an hour timescale) absorption (Perna \& Loeb 1998) and emission-line features (Böttcher et al. 1999; Ghisellini et al. 1999), such as those claimed to be seen in X-rays. On longer timescales, the GRB photoionization may lead to indicative recombination line features, which allow the identification of remnants of GRBs in nearby galaxies (Band \& Hartmann 1992; Perna et al. 2000, PRL in the following).

Photoionization of the ambient medium is well-studied in the case of supernovae, which are the cases most similar to GRBs.

\footnotetext{
* Based on observations collected at the European Southern Observatory, Chile (ESO Program 075.D-0771(A)).
}

For SN 1987A, IUE observations showed that the prominent UV lines started to increase simultaneously after 60-80days and stayed at a constant level until 400 days after the initial exciting supernova outburst (Fransson et al. 1989). After 400 days, most lines decreased quickly and reached the noise level by day 1500 (Sonneborn et al. 1997). Detailed modelling of the ionization zones and the line emission of the circumstellar gas, performed by Lundqvist \& Fransson (1996), allowed them to constrain the gas density.

In the case of a GRB, ionization by the prompt emission, the afterglow (photon field), and the blast wave (shock-ionization) will appear in addition to the SN component and will largely dominate. The blast wave is expected to influence the ionization state of the gas on timescales of hundreds to thousands of years after the burst (PRL). Therefore, for the purpose of our analysis, we can safely assume that photoionization is the dominating ionization mechanism. A high fraction of the energy (e.g. kinetic, magnetic) stored in the GRB-jet $\left(\sim 10^{52} \mathrm{erg}\right)$ is released in the afterglow. The X-ray/UV component of this radiation (with some contribution from the X-ray emission of the prompt phase) is the main one responsible for the ionization of the ambient medium. For typical GRB/afterglow luminosities, the size of the ionized region is on the order of $100 \mathrm{pc}$ for an ISM density of $\sim 1 \mathrm{~cm}^{-3}$ (PRL).

After being ionized, the gas then starts to cool on a timescale $t_{\text {cool }} \sim 10^{5}\left(T / 10^{5} \mathrm{~K}\right) /\left(n / \mathrm{cm}^{-3}\right) \mathrm{yr}$, where $T$ is the temperature of the gas and $n$ the electron density. Although cooling starts soon after the burst of radiation has passed through the medium, emission from the cooling gas increases during the early times 
after the GRB. If a region of radius $R$ is heated and ionized by the burst radiation, the maximum emission will occur after a time $t \sim R / c \sim 3 \mathrm{yr}(R / \mathrm{pc})$, due to light travel times from different parts of the region.

The strength and timescale of the recombination emission strongly depends on the ambient density. While the modelling of the broad-band SED of afterglows has led to densities in the range $1-10 \mathrm{~cm}^{-3}$, there are also observational indications of much higher densities: (i) observed variable X-ray lines (Watson et al. 2002; Reeves et al. 2003; Frontera et al. 2004) and continuum absorption (Lazzati \& Perna 2002) require densities of $\sim 10^{5}-10^{6} \mathrm{~cm}^{-3}$; (ii) some GRB afterglow data require a dense $\left(\sim 10^{4} \mathrm{~cm}^{-3}\right)$ shell around some nearby low-density media $\left(1-10 \mathrm{~cm}^{-3}\right)$ (Chevalier et al. 2004). The SN-GRB connection is now clearly proven for four GRBs (Galama et al. 1998; Hjorth et al. 2003; Stanek et al. 2003; Malesani et al. 2004; Pian et al. 2006), indicating the link between long-duration GRBs and the deaths of massive stars. Observations of massive, Wolf-Rayet (WR)-like stars have shown that they loose matter via strong stellar winds. A WR stellar wind, interacting with a circumstellar medium, leads to the formation of a shell (termination shock) whose density and radial extent depend on both the progenitor characteristics (i.e. mass loss rate, wind velocity), as well as on the density of the medium (e.g. Fryer et al. 2006).

An ionized shell of, say, density $\sim 10^{3} \mathrm{~cm}^{-3}$ and radial extent of a few parsecs will reach its peak emission on a timescale of a few years (due to the light travel times) and will cool on a timescale of tens of years. Therefore, the first few years after the burst are crucial for detecting cooling emission from these dense, compact shells produced by the wind termination shocks of the massive stars progenitors of (long) GRBs.

This Research Note reports a long-term monitoring, up to 6 years, of several strong emission lines and, in particular, of the OIII $\lambda 5007$ and the $\mathrm{H} \beta$ lines, whose ratio is an important discriminator of strongly photoionized, cooling gas as expected in GRB remnants (PRL). Our observations are described in Sect. 2, and the results are used in Sect. 3 to set constraints on the environment of GRB 990712. Further implications for studies of galaxy properties are discussed in Sect. 4, while Sect. 5 summarizes our findings.

\section{GRB 990712}

GRB 990712 was discovered by GRBM and WFC onboard BeppoSAX on July 12.69655 UT, 1999. The duration of the burst was $30 \mathrm{~s}$ and it was first localized by WFC at RA = 22:31:50, Dec $=-73: 24.4$ with an error radius of $2^{\prime}$ (Heise et al. 1999). Follow-up observations led to the discovery of the GRB afterglow. The redshift of the burst is $z=0.433$ (Vreeswijk et al. 2001a). Two different groups have found evidence of an SN bump from the optical lightcurve of the afterglow (Björnsson et al. 2001; Zeh et al. 2004). The BeppoSAX spectra of the prompt emission indicated a temporal emission feature located around $4.5 \mathrm{keV}$, which can be fit either with a Gaussian profile with a rest-frame energy of around $6.4 \mathrm{keV}$, which is consistent with an iron line, or with a blackbody spectrum with $k T \sim 1.3 \mathrm{keV}$ (Frontera et al. 2001). Since the iron line interpretation requires a very high-density environment that would obscure the afterglow, Frontera et al. (2001) prefer the thermal component interpretation that can be accounted for by the fireball model.

The host galaxy of GRB 990712 is one of the brightest GRB host galaxies, with $V=22.3 \mathrm{mag}$ and $R=21.8 \mathrm{mag}$ (Sahu et al. 2000). We obtained spectra of GRB 990712 on
Table 1. Log of observations.

\begin{tabular}{ccccc}
\hline \hline Date & Instrument & Grism & $\begin{array}{c}\text { Coverage } \\
(\mathrm{nm})\end{array}$ & $\begin{array}{c}\text { Exposure time } \\
(\mathrm{s})\end{array}$ \\
\hline 14 Jul. 1999 & FORS1 & 150I & $370-770$ & 2400 \\
11 Nov. 1999 & EFOSC & Gr6 & $400-800$ & 1800 \\
06 Jun. 2002 & FORS1 & 600R & $525-745$ & 4320 \\
17 Jul. 2004 & FORS2 & 600RI & $512-845$ & 2400 \\
05-06 Jul. 2005 & FORS2 & 300V & $445-850$ & 7200 \\
\hline
\end{tabular}

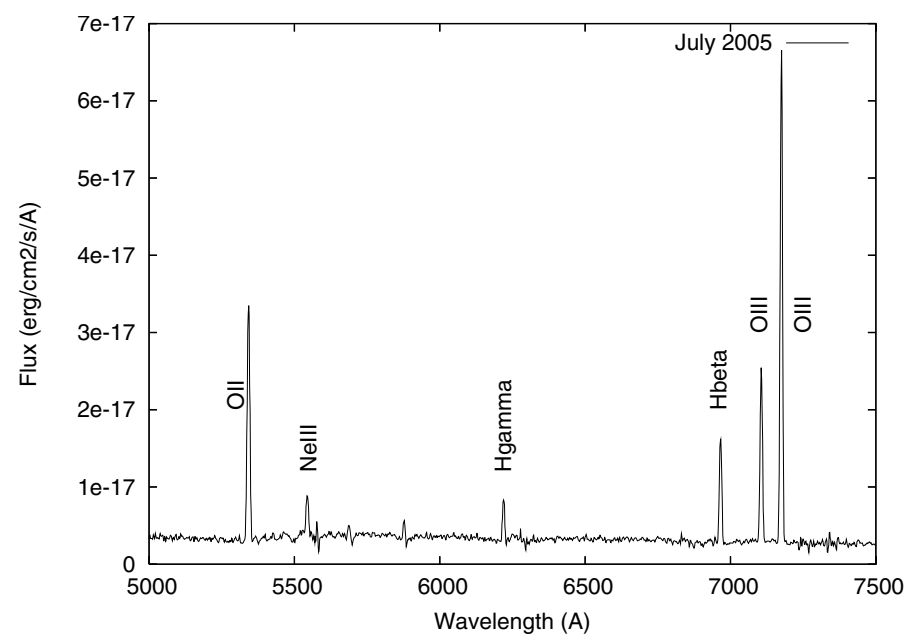

Fig. 1. Spectra of the host galaxy of GRB 990712 obtained in July 2005.

July 5 and 6, 2005, approximately 6 years after the GRB, using VLT/FORS2 under good seeing conditions $\left.(\sim 0)^{\prime} 6\right)$. The $300 \mathrm{~V} / \mathrm{GG} 435 \mathrm{grism} /$ filter was used with a slit width of $1{ }^{\prime \prime} 0$. The total exposure time was $2 \mathrm{~h}(4 \times 30 \mathrm{~min})$. The spectra were reduced using standard IRAF routines and calibrated using standard star G158-100 observed on July 5, 2005 with the same grism/filter. The flux calibration was further validated by folding the spectrum with the FORS $R$-band filter curve and comparing the obtained magnitude with that of the host galaxy given in Christensen et al. (2004). We corrected the spectra for foreground extinction of $E(B-V)=0.03$ (Schlegel et al. 1998). The line fluxes were determined by fitting a Gaussian to the line using the SPLOT task of IRAF. The continuum level was determined locally.

The line fluxes are compared with those derived using VLT archival data of observations obtained on July 14, 1999 (PI: Galama), November 11, 1999 (PI: Courbin), June 6, 2002 (PI: Mirabel), and on July 17, 2004 (PI: Le Floc'h) (see Table 1). The spectra were treated in an identical way and calibrated using standard stars EG 274 (Sep. 15, 1999), LTT 377 (on Dec. 6, 1999 and on July 15, 2004), and LTT 3854 (June 5, 2002) for July 1999, November 1999, July 2004, and June 2002 data, respectively. July 1999 data was further flux-calibrated using the afterglow brightness at that time taken from Sahu et al. (2000). The flux values we obtained are consistent with the published values obtained by flux-calibrating the same data using the afterglow brightness extrapolated in time (Vreeswijk et al. 2001a). The previously unpublished 2002 and 2004 fluxes were similarly validated as in the case of 2005 spectra. The estimated error in the flux calibration is about $10 \%$ for all data. The line fluxes are shown in Table 2. The flux errors in Table 2 only include the uncertainities in the continuum level. Figure 1 shows our spectrum 
Table 2. Emission line fluxes.

\begin{tabular}{cccccc}
\hline \hline Line & \multicolumn{4}{c}{ Flux $\left(10^{-16} \mathrm{erg} \mathrm{s}^{-1} \mathrm{~cm}^{-2}\right)$} \\
& at day 1.5 & at day 123 & at day 1060 & at day 1832 & at day 2185 \\
\hline$[\mathrm{O} \mathrm{II}](\lambda 3727)$ & $3.52 \pm 0.15$ & & $3.66 \pm 0.20$ & & $3.40 \pm 0.60$ \\
{$[\mathrm{NeIII}](\lambda 3869)$} & $0.5 \pm 0.1$ & & $0.55 \pm 0.07$ & $0.59 \pm 0.05$ & $0.50 \pm 0.05$ \\
$\mathrm{H} \gamma(\lambda 4340)$ & $0.3 \pm 0.1$ & & $0.53 \pm 0.12$ & $0.46 \pm 0.05$ & $0.46 \pm 0.05$ \\
$\mathrm{H} \beta(\lambda 4861)$ & $1.15 \pm 0.15$ & $1.30 \pm 0.15$ & $1.29 \pm 0.09$ & $1.34 \pm 0.04$ & $1.33 \pm 0.05$ \\
{$[\mathrm{OIII}](\lambda 4959)$} & $2.25 \pm 0.15$ & $2.33 \pm 0.18$ & $2.10 \pm 0.15$ & $2.18 \pm 0.08$ & $2.22 \pm 0.08$ \\
{$[\mathrm{OIII}](\lambda 5007)$} & $6.15 \pm 0.15$ & $6.17 \pm 0.25$ & $6.00 \pm 0.10$ & $5.97 \pm 0.08$ & $6.08 \pm 0.08$ \\
\hline
\end{tabular}

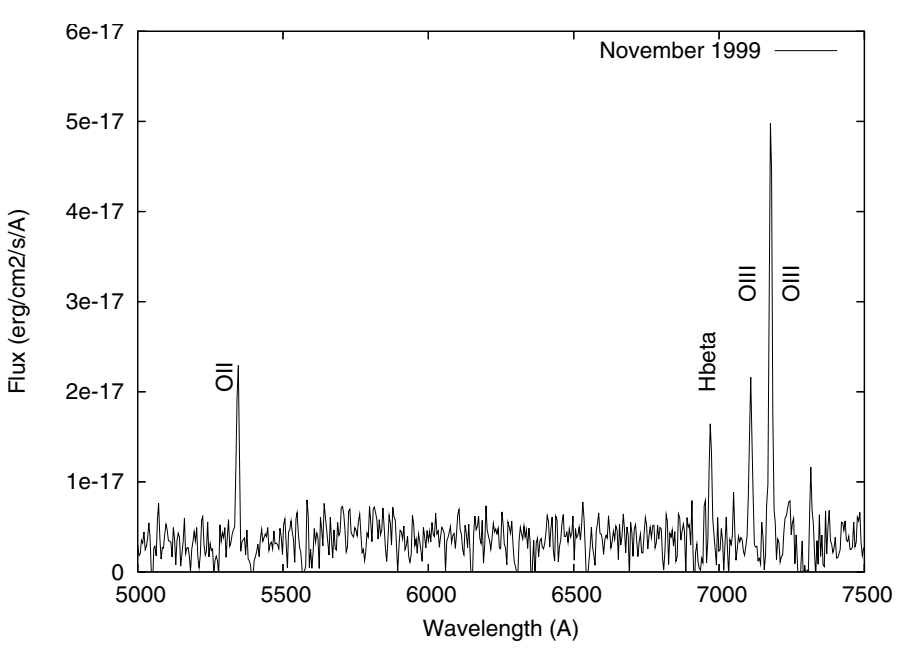

Fig. 2. Spectra of the host galaxy of GRB 990712 obtained in November 1999.

from July 2005 and Fig. 2 shows the spectrum from November 1999, which has the worst signal-to-noise ratio.

All of the observed lines, which are [O II], [Ne III], $\mathrm{H} \gamma, \mathrm{H} \beta$, and [O III] $(\lambda \lambda 4959,5007)$ lines, are observed to have constant fluxes over $\sim 6$ years after the burst (see Fig. 3 ). To overcome the difficulty of comparing line fluxes from different spectra obtained with different settings, i.e. instrument, grism, night conditions, etc., we derive our results on the circumburst environment based particularly on the [O III] $\lambda 5007$ and the $\mathrm{H} \beta$ lines. These are close enough in wavelength space to overcome the possible effects of different flux calibrations, and at the same time, as discussed below, they are especially strong discriminators of photoionization vs. collisional ionization.

\section{The circumburst environment}

A distinctive signature of photoionized gas is a high [O III] $\lambda 5007 / \mathrm{H} \beta$ ratio $(\gtrsim 5)$, which is not generally produced in steady shocks for solar abundances (Shull \& McKee 1979). This ratio increases with the ionization parameter and, for typical $\mathrm{X}$-ray/UV photoionizing fluxes of GRBs and their afterglows, it reaches a value on the order of 100 (PRL). Our observations at about 6 years after the burst, as well as the previous ones at earlier times, show that [O III] $\lambda 5007 / \mathrm{H} \beta \sim 4.6$ in the host of GRB 990712. Combined with the $\log ([\mathrm{O} \mathrm{II}] / \mathrm{H} \beta)$ value of $0.4 \pm 0.1$, this value is rather typical of an HII galaxy, as can be seen by comparison with the sample of emission line galaxies at redshifts $0 \lesssim z \lessgtr 0.3$ from the Canada-France redshift survey (Rola et al. 1997; see also Vreeswijk et al. 2001a). Therefore, the constraints that we are able to put on the close environment of

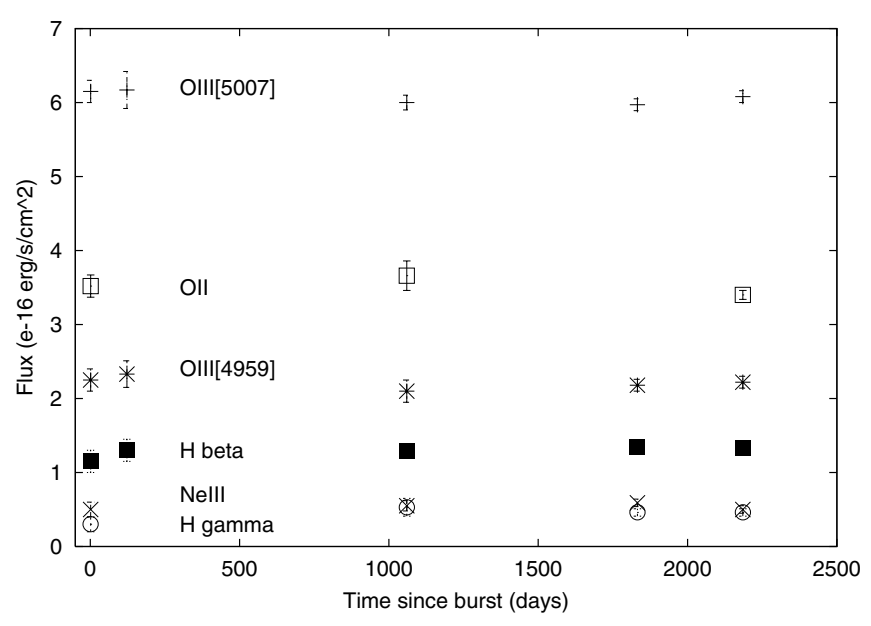

Fig. 3. [Ne III], H $\gamma, \mathrm{H} \beta$, [O II], and [O III] 4959, 5007 line flux light curves for GRB 990712. Error bars are smaller than the symbol size, if not visible.

GRB 990712 can be derived from the lack of contribution from photoionized gas to the brightest expected line ([O III] 15007) from the cooling gas, within the observational uncertainties. The measured flux of this line is $\sim 6 \times 10^{-16} \mathrm{erg} \mathrm{s}^{-1} \mathrm{~cm}^{-2}$ to within $\sim 10 \%$ uncertainty (see Table 2 ). At a redshift of 0.433 , this flux corresponds to a luminosity of $\sim 3.8 \times 10^{41} \mathrm{erg} \mathrm{s}^{-1}$ for a LCDM cosmology with Hubble parameter $h=0.73$ (Spergel et al. 2006). Since the luminosity is constant to within the $10 \%$ uncertainty value of $\sim 3 \times 10^{40} \mathrm{erg} \mathrm{s}^{-1}$, the contribution from the cooling GRB remnant cannot be larger than this value at any given time during the observation window.

Numerical simulations of cooling GRB remnants (PRL) show that the radiation flux from a typical GRB and its afterglow ionizes a region on the order of $\sim 100 \mathrm{pc}$ for an ISM density of $\sim 1 \mathrm{~cm}^{-3}$. For a gas of solar metallicity, the corresponding luminosity of the [O III] $\lambda 5007$ line from the cooling gas is found to reach a value of $\sim 10^{38} \mathrm{erg} \mathrm{s}^{-1}$ over a time $t_{\text {cool }} \sim$ a few $\times$ $10^{4} \mathrm{yr}$ (see Fig. 3 in PRL; the details of the computation of the cooling radiation can also be found in PRL). The brightness of the line scales with $n n_{\mathrm{e}}$, where $n_{\mathrm{e}}$ is the electron density. For a gas metallicity not too far from solar (so that the particle number is dominated by $\mathrm{H}$ ) and a highly ionized gas, one has $n_{\mathrm{e}} \sim n$, and the line luminosity can be scaled, to a first approximation, as ${ }^{1} \sim 10^{38}\left(\mathrm{n} / \mathrm{cm}^{-3}\right)^{2}\left(R_{\mathrm{e}} / 100 \mathrm{pc}\right)^{3} \mathrm{erg} \mathrm{s}^{-1}$. Our observations, up to 6 years after the burst, allow us to probe an

1 Note however that, as the density increases, the maximum size of the region that can be ionized will decrease. Furthermore, note that the details of the rise time also depend on the extent of the beaming of the ionizing radiation, but we do not worry about secondary effects here since the data only allow us to set upper limits. 
emitting region, $R_{\mathrm{e}}$, of at most $2 \mathrm{pc}$ in size, due to light delay effects, as discussed in Sect. 1. Since no flux variation has been observed within the $10 \%$ flux error (corresponding to a luminosity of about $3 \times 10^{40} \mathrm{erg} \mathrm{s}^{-1}$ ), we deduce that the line luminosity due to the cooling gas has to be below this level, i.e.

$$
\begin{aligned}
L_{5007} \sim & 10^{38}\left(\mathrm{n} / \mathrm{cm}^{-3}\right)^{2} \\
& \times\left(R_{\mathrm{e}} / 100 \mathrm{pc}\right)^{3} \mathrm{erg} \mathrm{s}^{-1} \lesssim 3 \times 10^{40} \mathrm{erg} \mathrm{s}^{-1},
\end{aligned}
$$

which yields the limit on the density $n \lesssim 6 \times 10^{3} \mathrm{~cm}^{-3}$ for $R_{\mathrm{e}}=2 \mathrm{pc}$. This limit can be used to constrain the range of allowed parameters for the GRB host and ISM densities. For example, Fryer et al. (2006) find that, for a progenitor star with mass loss $\dot{M}=10^{-5} M_{\odot} \mathrm{yr}^{-1}$ and wind velocity $\sim 1000 \mathrm{~km} \mathrm{~s}^{-1}$, expanding in a medium of density in the range $\sim 10^{3}-10^{4} \mathrm{~cm}^{-3}$, the inner radius of the shell is on the order of tenths of a par$\mathrm{sec}$, and the outer radius is $>2 \mathrm{pc}$ (with the shell density being on the order of the ISM density). These high shell densities, filling a region up to the observed emitting volume of $\sim 2 \mathrm{pc}$, are not favored by our observations, since they would likely result in a variable [O III] $\lambda 5007$ flux over the 6 years of observations. On the other hand, termination-shock shells produced from the impact of the wind with a lower-density medium are consistent with this lack of variability. Also note that, given the 6 year timescale of the observations, termination shocks located at distances $\gtrsim 2$ pc cannot be ruled out by the currently available data. Generally speaking, observations at longer timescales allow one to probe shell termination-shocks over a wider range of distances from the GRB progenitor star. However, since more distant termination shocks are generally associated with lower ISM densities (and hence a lower luminosity of the [O III] $\lambda 5007$ line), in order to separate the eventual contribution of this line due to the cooling gas in the close GRB environment, from that due to the host galaxy itself, a higher signal-to-noise ratio in the observations as well as a consistent set of observations (i.e. observations obtained with the same instrument and settings) is necessary.

\section{Star formation rate and metallicity}

The star formation rate (SFR) of the host galaxy of GRB 990712 has been previously calculated based on line fluxes and ultraviolet flux, and also an upper limit has been derived based on radio non-detection (Hjorth et al. 2000; Vreeswijk et al. 2001a,b; Christensen et al. 2004). Using the line fluxes of our July 2005 spectrum and the same method used by Vreeswijk et al. (2001a), we calculated $S F R_{\mathrm{OII}}=2.8_{-0.9}^{+0.4} M_{\odot} / \mathrm{yr}$ and $A_{\mathrm{V}}=$ $1.7_{-0.8}^{+0.9}$ mag based on the $\mathrm{H} \gamma / \mathrm{H} \beta$ ratio. Our $A_{\mathrm{V}}$ value is, on average, lower than the $A_{\mathrm{V}}=3.4_{-17}^{+2.4}$ derived by Vreeswijk et al. (2001a); therefore, our extinction-corrected SFR value $\left(S F R_{\text {OIIcorr }}=10_{-6}^{+15} M_{\odot} / \mathrm{yr}\right)$ is also lower than their calculation. Nonetheless, both $A_{\mathrm{V}}$ and the extinction-corrected SFR agree with the Vreeswijk et al. (2001a,b) values within the errors.

We did not detect the [O III] $\lambda 4363$ line that is necessary to determine the electron temperature $T_{\mathrm{e}}$ by means of lines (like [O III], [Ne III], etc.) from high ionization-zone elements. Therefore, we cannot estimate the oxygen and neon abundance based on the electron temperature. However, we can still estimate the oxygen abundance based on the ratio $R_{32}=([\mathrm{O}$ II $] \lambda 3727+[\mathrm{O}$ III $] \lambda \lambda 4959,5007) / \mathrm{H}_{\beta}$. Kewley \& Dopita (2002) suggest using the relation given by Zaritsky et al. (1994) to obtain an estimate of the oxygen abundance. Using the formulae given in Zaritsky et al. (1994), we obtain $\log (\mathrm{O} / \mathrm{H})=-3.7 \pm 0.1$. However, that formula is calibrated for metal rich galaxies and overestimates the metallicity for values of $\log (\mathrm{O} / \mathrm{H})<-3.5$ (see Kobulnicky et al. 1999; Kewley \& Dopita 2002). Therefore we used Eq. (16) in Kobulnicky \& Kewley (2004), which is adapted from the relation given by Kewley \& Dopita (2002) and parameterized for the lower metallicity branch $(\log (\mathrm{O} / \mathrm{H})<-3.6)$. The result is $\log (\mathrm{O} / \mathrm{H})=$ $-3.7 \pm 0.1$, which is the same as our initial estimate using the relation given by Zaritsky et al. (1994). Similarly, Vreeswijk et al. (2001a) obtained $-3.7 \pm 0.4$ for $\log (\mathrm{O} / \mathrm{H})$, which agrees with our estimate. The oxygen abundance we obtained is just a bit lower than the solar value $(\log (\mathrm{O} / \mathrm{H})=-3.34$; e.g. Asplund et al. 2005). Therefore our assumption that the metallicity of the gas near the GRB is not far from solar is reasonable in our derivation of the [O III] line luminosity of the cooling gas.

It should be noted that, in the context of GRBs, studying the photoionization signatures of cooling gas not only helps in understanding the nature of the GRB progenitor star, but it also helps reduce possible biases in the determinations of two important properties of the GRB host galaxy, such as the SFR and the metallicity. In fact, SFR and metallicity calculations based on emission lines generally rely on observations obtained at a single epoch. However, in order to properly assess the possible level of contamination by the cooling GRB remnant, multiple epochs of observations spanned over a long timescale are necessary.

\section{Summary}

We have presented the results of the spectral analysis of the host galaxy of GRB 990712. With the last set of observations taken about 6 years after the burst, this is the longest time coverage for a GRB host galaxy to date. Though we do not detect line variations, timescales of a few years are important for detecting cooling radiation from the heated shells produced by the wind termination shocks of the massive star progenitors of the GRBs. For the case of the GRB 990712 host, the lack of time variability in the [O III] $\lambda 5007$ line, combined with the $\$ 5$ ratio of the $[\mathrm{O}$ III $] \lambda 5007 / \mathrm{H} \beta$ lines, has allowed us to set an upper limit to the contribution from the cooling gas. This limit, in turn, could be used to constrain the allowed range of densitites within a region of about 2 pc surrounding the burst. We have therefore shown how this type of observation provides a useful complement to the studies of the close environment of GRB progenitors and, therefore, can help reconstruct the characteristics of the GRB progenitor star.

Finally, we have pointed out how, if a substantial contamination to the galaxy spectra is provided by the GRB cooling radiation, inferences of the SFR and metallicity that are drawn from measurements of line ratios can be biased. To be able to assess the degree of this contamination, long-term monitoring of the GRB host galaxies is necessary.

Acknowledgements. We thank Arne Rau and Markus Böttcher for insightful comments and discussions that contributed to the early stages of this work, and John Raymond for very useful comments on the manuscript. A.K.Y. acknowledges support from the International Max-Planck Research School (IMPRS) on Astrophysics. R.P. acknowledges support from NASA under grant NNG05GH55G and from the NSF under grant AST 0507571.

\section{References}

Asplund, M., Grevesse, N., \& Sauval, A. J. 2005, ASPC, 336, 25 Band, D. L., \& Hartmann, D. H. 1992, ApJ, 386, 299

Björnsson, G., Hjorth, J., Jakobsson, P., et al. 2001, ApJ, 552, L121

Böttcher, M., Dermer, C. D., Crider, A. W., \& Liang, E. P. 1999, A\&A, 343, 111 
Chevalier, R., Li, Z.-Y., \& Fransson, C. 2004, ApJ, 606, 369

Christensen, L., Hjorth, J., Gorosabel, J., et al. 2004, A\&A, 413, 121

Fransson, C., Cassatella, A., Gilmozzi, R., et al. 1989, ApJ, 336, 429

Frontera, F., Amati, L., Vietri, M., et al. 2001, ApJ, 550, L47

Frontera, F., Amati, L., Lazzati, D., et al. 2004, ApJ, 614, 301

Fryer, C. L., Rockefeller, G., \& Young, P. A. 2006, ApJ, 647, 1269

Galama, T. J., Vreeswijk, P. M., van Paradijs, J., et al. 1998, Nature, 395, 670

Ghisellini, G., Haardt, F., Campana, S., et al. 1999, ApJ, 517, 168

Heise, J., in't Zand, J., Tarei, G., et al. 1999, IAU Circ., 7221

Hjorth, J., Holland, S., Courbin, F., et al. 2000, ApJ, 534, L147

Hjorth, J., Sollerman, J., Mller, P., et al. 2003, Nature, 423, 847

Kewley, L. J., \& Dopita, M. A. 2002, ApJS, 142, 35

Kobulnicky, H. A., Kennicutt, R. C., \& Pizagno, J. L. 1999, ApJ, 514, 544

Kobulnicky, H. A., \& Kewley, L. J. 2004, ApJ, 617, 240

Lazzati, D., \& Perna, R. 2002, MNRAS, 330, 383

Lundqvist, P., \& Fransson, C. 1996, ApJ, 464, 924

Malesani, D., Tagliaferri, G., Chincarini, G., et al. 2004, ApJ, 609, 5

Perna, R., \& Loeb, A. 1998, ApJ, 501, 467
Perna, R., Raymond, J., \& Loeb, A. 2000, ApJ, 533, 658

Pian, E., Mazzali, P. A., Masetti, N., et al. 2006 [arXiv: astro-ph/0603530]

Rees, M. J., \& Meszaros, P. 1992, MNRAS, 258, 41

Rees, M. J., \& Meszaros, P. 1998, ApJ, 496, L1

Reeves, J. N., Watson, D., Osborne, J. P., Pounds, K. A., \& O’Brien, P. T. 2003 , A\&A, 403, 463

Rola, C. S., Terlevich, E., \& Terlevich, R. J. 1997, MNRAS, 289, 419

Sahu, K. C., Vreeswijk, P., Bakos, G., et al. 2000, ApJ, 540, 74

Schlegel, D. J., Finkbeiner, D. P., \& Davis, M. 1998, ApJ, 500, 525

Shull, J. M., \& McKee, C. F. 1979, ApJ, 227, 131

Spergel, D. N., Bean, R., Dore', O., et al. 2006 [arXiv : astro-ph/0603449]

Sonneborn, G., Fransson, C., Lundqvist, P., et al. 1997, ApJ, 477, 848

Stanek, K. Z., Matheson, T., Garnavich, P. M., et al. 2003, ApJ, 591, L17

Vreeswijk, P., Fruchter, A., Kaper, L., et al. 2001a, ApJ, 546, 672

Vreeswijk, P., Fender, R. P., Garrett, M. A., et al. 2001b, A\&A, 380, L21

Watson, D., Reeves, J. N., Osborne, J., et al. 2002, A\&A, 393, L1

Zaritsky, D., Kennicutt, R. C., \& Huchra, J. P. 1994, ApJ, 420, 87

Zeh, A., Klose, S., \& Hartmann, D. H. 2004, ApJ, 609, 952 